\title{
Generic scalar potentials in geometric scalar gravity
}

\author{
Nahomi Kan $^{1} \cdot$ Kiyoshi Shiraishi ${ }^{2}($ i)
}

Received: 7 November 2015/Accepted: 23 December 2015/Published online: 27 January 2016

(c) The Author(s) 2016. This article is published with open access at Springerlink.com

\begin{abstract}
We discuss a generic form of the scalar potential appearing in the geometric scalar theory of gravity. We find the conditions on the potential by considering weak and strong gravity. The modified black hole solutions are obtained for generic potentials and the inverse problems on a black hole and on a spherical body ('pseudogravastar') are investigated.
\end{abstract}

Keywords Modified gravity $\cdot$ Black holes $\cdot$ Newtonian approximation · Compact objects

\section{Introduction}

General relativity (GR) is a unique theory of gravity which passes various experimental tests, at least to our present knowledge. The modifications of GR are, however, studied by many researchers for the reason that they may resolve the cosmological riddles about dark matter and/or dark energy [1-3].

In recent years, Novello and collaborators advocated a novel theory of gravity, called geometric scalar gravity (GSG) [4-8]. In this theory, the dynamic field is a single scalar field, but a normalized derivative of the scalar field expresses a part of the dynamical metric, as well as the

Kiyoshi Shiraishi

shiraish@yamaguchi-u.ac.jp

Nahomi Kan

kan@gifu-nct.ac.jp

1 National Institute of Technology, Gifu College, Motosu-shi, Gifu 501-0495, Japan

2 Graduate School of Science and Engineering, Yamaguchi University, Yamaguchi-shi, Yamaguchi 753-8512, Japan scalar field itself. Therefore, it is possible to avoid the difficulty in old scalar gravitation theories not including derivative terms [9-12]. ${ }^{1}$

Novello is also insisting [13] that more scalar degrees of freedom are necessary to explain the gravitational field around the spinning source more practically. However, it is a challenging problem for theorists to clarify how GSG, as the simplest model or as a case where many scalar degrees of freedom freeze, can be adjusted to realistic gravity.

As a modified theory of gravity, mimetic gravity [14] has been proposed, where a normalized scalar derivative term is also used for the dynamical metric. If one would construct such a modified theory of gravity from tensor and scalar fields, it is worth examining the behavior of the scalar model in the limit of a few degree of freedom and pursuing how many aspects can be found through the study of the simplest theory. Based on the research, we could advance the study of theories including a vector field, such as $\mathrm{TeVeS}$ [15], and more complicated theories.

Novello and collaborators chose the specific form for the potential of the scalar field from which the Schwarzschild spacetime is derived as a spherically symmetric vacuum solution, and further advanced towards the discussion of the (exotic) cosmology based on it [4-8]. However, because the dynamics of gravity in GSG is completely different from GR, so we need not require perfect coincidence of the solution with an exact solution of GR. Actually, we have to check observational validity through description of the parametrized post-Newton treatment of the weak gravitational field (see for example, [16, 17]). Therefore, there may be a finite ambiguity in the form of the permissible potential. At first, we pay attention to this

${ }^{1}$ For older many works, please see the references therein. 
point and expose the necessary condition that the potential should satisfy.

Another important issue of the non-linear theory of gravity is avoidance of the singularity. A singular point is concealed by a horizon of a black hole in GR. There is room of argument for whether GSG can also be used without a correction in the strong gravity, but we wish to know what kind of the potential provides a spherically symmetric black hole solution with a horizon in GSG.

From these two discussions about weak and strong gravity, we consider possible forms of the scalar potential and some restrictions on GSG.

This paper is organized as follows. In "A brief review of GSG", we begin by reviewing GSG to make this paper selfcontained. Then, we consider a spherical gravitational field in GSG and clarify the condition on the relation between the scalar potential and parametrized post-Newtonian description in "A static spherical solution and the post-Newtonian parameters". A possible singularity in the spherical vacuum solution in GSG is studied in "A static spherical vacuum solution and singularity". The condition on the scalar potential for emerging a horizon is revealed. In "Inverse problems in GSG", the potential which leads to a slightly modified Schwarzschild solution is obtained, such as an inverse problem. In this section, we also consider the spherical body and investigate behaviors of the energy density and the pressure of the static fluid for a given de Sitter-like spacetime as another inverse problem. Finally, we give summary and prospects in "Summary and prospects".

\section{A brief review of GSG}

In this section, we briefly review GSG [4] to make the present paper self-contained. In GSG, the physical metric $q_{\mu \nu}$ is described by a scalar field $\Phi$ as

$q_{\mu \nu}=e^{2 \Phi}\left[\eta_{\mu v}-\frac{e^{-4 \Phi} V(\Phi)-1}{e^{-4 \Phi} V(\Phi)} \frac{\partial_{\mu} \Phi \partial_{\nu} \Phi}{w}\right]$,

where

$w \equiv \eta^{\mu v} \partial_{\mu} \Phi \partial_{v} \Phi$

and $\eta_{\mu v}$ is a flat Minkowski metric with the signature $(+---)$. The inverse of the metric is then written as

$q^{\mu \nu}=e^{-2 \Phi}\left[\eta^{\mu v}+\frac{e^{-4 \Phi} V(\Phi)-1}{w} \eta^{\mu \rho} \eta^{v \sigma} \partial_{\rho} \Phi \partial_{\sigma} \Phi\right]$,

and note that

$\sqrt{-q}=\sqrt{-\operatorname{det} q_{\mu \nu}}=\frac{e^{6 \Phi}}{\sqrt{V(\Phi)}} \sqrt{-\eta}$.

We should also note that $q^{\mu v} \partial_{v} \Phi=e^{-6 \Phi} V(\Phi) \eta^{\mu v} \partial_{v} \Phi$.
We call $V(\Phi)$ the (scalar) potential in the present paper. This function determines the dynamics of the scalar $\Phi$, and only the property of gravity constrains its concrete form, as far as we do not assume any symmetry argument.

The action governing the dynamics of the scalar field $\Phi$ with a potential $V(\Phi)$ is given by

$S_{\Phi}=\frac{1}{\kappa} \int \sqrt{-q} \sqrt{V(\Phi)} q^{\mu v} \partial_{\mu} \Phi \partial_{v} \Phi \mathrm{d}^{4} x$

where $\kappa$ is a constant. The variation of the action with respect to the scalar $\Phi$ is calculated as

$\delta S_{\Phi}=-\frac{2}{\kappa} \int \sqrt{-q} \sqrt{V(\Phi)}(\square \Phi) \delta \Phi \mathrm{d}^{4} x$

where

$$
\begin{aligned}
\square \Phi & \equiv \frac{1}{\sqrt{-q}} \partial_{\mu}\left(\sqrt{-q} q^{\mu v} \partial_{v} \Phi\right) \\
& =e^{-6 \Phi} V(\Phi)\left[\frac{1}{\sqrt{-\eta}} \partial_{\mu}\left(\sqrt{-\eta} \eta^{\mu v} \partial_{v} \Phi\right)+\frac{w}{2} \frac{\mathrm{d}}{\mathrm{d} \Phi} \ln V(\Phi)\right]
\end{aligned}
$$

The action for matter fields is presumed as

$S_{m}=\int \sqrt{-q} \mathcal{L}_{m} \mathrm{~d}^{4} x$,

and its variation with respect to the metric is written as follows:

$\delta S_{m}=-\frac{1}{2} \int \sqrt{-q} T^{\mu v} \delta q_{\mu \nu} \mathrm{d}^{4} x$

where

$T_{\mu \nu} \equiv \frac{2}{\sqrt{-q}} \frac{\partial \sqrt{-q} \mathcal{L}_{m}}{\partial q^{\mu \nu}}$

is the energy-momentum tensor of matter fields. Some manipulation leads to the following result on the variation with respect to the scalar $\Phi$ :

$T^{\mu v} \delta q_{\mu v}=\left[2 T+\left(4-\frac{1}{V} \frac{\mathrm{d} V}{\mathrm{~d} \Phi}\right) E\right] \delta \Phi-2 C^{\lambda} \partial_{\lambda} \delta \Phi$

where

$$
\begin{gathered}
T=T^{\mu v} q_{\mu v}, \quad E=\frac{T^{\mu v} \partial_{\mu} \Phi \partial_{v} \Phi}{\Omega}, \\
C^{\lambda}=\frac{e^{-4 \Phi} V-1}{\Omega}\left(T^{\lambda v}-E q^{\lambda v}\right) \partial_{v} \Phi,
\end{gathered}
$$

with

$\Omega \equiv q^{\mu v} \partial_{\mu} \Phi \partial_{v} \Phi=e^{-6 \Phi} V w$.

We then obtain the variation of the action for matter fields with respect to $\Phi$ as 
$\delta S_{m}=-\int\left[T+\left(2-\frac{1}{2 V} \frac{\mathrm{d} V}{\mathrm{~d} \Phi}\right) E+\nabla_{\lambda} C^{\lambda}\right] \delta \Phi \sqrt{-q} \mathrm{~d}^{4} x$,

where

$\nabla_{\lambda} C^{\lambda} \equiv \frac{1}{\sqrt{-q}}\left(\partial_{\lambda} \sqrt{-q} C^{\lambda}\right)$

Now we define the total action of the gravitating system as $S=S_{\phi}+S_{m}$.

The equation derived from this total action can be written as

$\sqrt{V(\Phi)} \square \Phi=\kappa \chi$

where

$\chi=-\frac{1}{2}\left[T+\left(2-\frac{1}{2 V} \frac{\mathrm{d} V}{\mathrm{~d} \Phi}\right) E+\nabla_{\lambda} C^{\lambda}\right]$.

Let us consider the Newtonian limit. The gravitating source can be estimated as

$T_{00} \approx \rho$,

where $\rho$ is the energy density and the other components of the energy-momentum tensor $T_{\mu \nu}$ can be neglected.

Novello et al. then assumed

$q_{00}=e^{2 \Phi} \approx 1+2 \Phi_{N}$,

where $\Phi_{N}$ denotes the static Newtonian potential. The other elements of the metric tensor are taken as those of the Minkowski metric.

We would like to consider the case with the non-zero asymptotic value of the scalar field $\Phi_{\infty}$. The redefinition of the time coordinate yields the metric component $q_{00}=$ $e^{2 \Phi_{\infty}} \rightarrow 1$ in the asymptotic region. In this case, since $\left|\Phi-\Phi_{\infty}\right| \approx\left|\Phi_{N}\right| \ll 1$, we find that the dynamical equation (2.17) can be approximated as

$$
\sqrt{V\left(\Phi_{\infty}\right)} \nabla^{2} \Phi=\frac{\kappa}{2} \rho
$$

Thus, if we set, with the definition of the Newton constant G

$$
\frac{\kappa}{\sqrt{V\left(\Phi_{\infty}\right)}}=8 \pi G
$$

we have a correct Newtonian limit.

\section{A static spherical solution and the post-Newtonian parameters}

Novello et al. considered a static spherical solution in GSG [4]. We are interested in generic spherically symmetric solutions of this theory. We start with their argument in this section. First, they considered the flat metric in the spherical coordinates,

$\eta_{\mu \nu} \mathrm{d} x^{\mu} \mathrm{d} x^{\nu}=\mathrm{d} T^{2}-\mathrm{d} R^{2}-R^{2} \mathrm{~d} \Omega^{2}$

with $\mathrm{d} \Omega^{2}$ is the line element on a unit sphere. Because the spherical symmetry enforces that $\Phi=\Phi(R)$, the physical line element is given as

$\mathrm{d} s^{2}=q_{\mu \nu} \mathrm{d} x^{\mu} \mathrm{d} x^{v}=e^{2 \Phi} \mathrm{d} T^{2}-\frac{e^{6 \Phi}}{V(\Phi)} \mathrm{d} R^{2}-e^{2 \Phi} R^{2} \mathrm{~d} \Omega^{2}$

Now, defining $t \equiv e^{\Phi_{\infty}} T$ and converting the radial coordinate to $r \equiv e^{\Phi} R$, we find

$\mathrm{d} s^{2}=B(r) \mathrm{d} t^{2}-A(r) \mathrm{d} r^{2}-r^{2} \mathrm{~d} \Omega^{2}$,

where

$B(r)=e^{2\left(\Phi-\Phi_{\infty}\right)}$,

$A(r)=\frac{e^{4 \Phi}}{V(\Phi)}\left(1-r \frac{\mathrm{d} \Phi}{\mathrm{d} r}\right)^{2}$

To obtain an asymptotically flat spacetime, we assume

$\lim _{r \rightarrow \infty} \Phi(r)=\Phi_{\infty}, \quad \lim _{r \rightarrow \infty} r \Phi^{\prime}(r)=0, \quad e^{-4 \Phi_{\infty}} V\left(\Phi_{\infty}\right)=1$,

where $\Phi^{\prime}(r)$ is the first derivative of $\Phi(r)$.

The equation of motion in vacuum $\square \Phi=0$ implies

$\frac{\mathrm{d}}{\mathrm{d} r}\left[\sqrt{\frac{B(r)}{A(r)}} r^{2} \frac{\mathrm{d} \Phi}{\mathrm{d} r}\right]=0$.

Novello et al. [4] insisted that the form for $V(\Phi)$ is chosen so as to realize the exact Schwarzschild solution. So, they chose

$\Phi_{\infty}=0, \quad V(\Phi)=V_{\mathrm{N}}(\Phi)=\frac{1}{4} e^{2 \Phi}\left(1-3 e^{2 \Phi}\right)^{2}$.

This criterion for the choice of $V(\Phi)$ is too severe for a model of weak gravity, we think. The Newtonian limit has been examined in the previous section. We study here the post-Newtonian limit. The possible asymptotic behavior determines the form of the scalar field as

$\Phi_{N} \equiv \Phi-\Phi_{\infty}=-\frac{G M}{r}-g \frac{G^{2} M^{2}}{r^{2}}+O\left((G M / r)^{3}\right)$

where $M$ is the gravitational mass and $g$ is a constant of dimensionless. The potential $V(\Phi)$ is assumed up to the linear order in $\Phi_{N}$, corresponding to the perturbative regime and we set

$e^{-4 \Phi} V(\Phi)=1+4 k \Phi_{N}+O\left(\Phi_{N}^{2}\right)$. 
Then the metric components $B(r)$ and $A(r)$ are expressed in terms of the parameters as

$$
\begin{aligned}
& B(r)=1-\frac{2 G M}{r}+\frac{2(1-g) G^{2} M^{2}}{r^{2}}+O\left((G M / r)^{3}\right), \\
& A(r)=1+\frac{2(2 k-1) G M}{r}+O\left((G M / r)^{2}\right) .
\end{aligned}
$$

and we then find that

$$
\sqrt{\frac{B(r)}{A(r)}} r^{2} \frac{\mathrm{d} \Phi}{\mathrm{d} r}=G M+\frac{2(g-k) G^{2} M^{2}}{r}+O\left(G M(G M / r)^{2}\right) .
$$

Therefore up to the order in the present consideration, the combination of the dynamic Eqs. (3.7) and (3.13) tells us $g=k, \quad$ so we obtain $B(r)=1-\frac{2 G M}{r}+\frac{2(1-k) G^{2} M^{2}}{r^{2}}+O\left((G M / r)^{3}\right)$.

According to the metric form with two post-Newtonian parameters $\beta$ and $\gamma[16,17]$

$B(r)=1-\frac{2 G M}{r}+\frac{2(\beta-\gamma) G^{2} M^{2}}{r^{2}}+O\left((G M / r)^{3}\right)$,

$A(r)=1+\frac{2 \gamma G M}{r}+O\left((G M / r)^{2}\right)$,

we can express two of the post-Newtonian parameters $\beta$ and $\gamma$ by using the linear coefficient $k$ in $V(\Phi)$ as

$\beta=k, \quad \gamma=2 k-1$.

For the experimentally viable values $\beta=\gamma=1[16,17]$, we should take $k=1$. Of course, the expansion of the potential chosen by Novello et al. (3.8) shows that it is in the case.

To summarize, we should select the form of the scalar potential $V(\Phi)$ as

$$
e^{-4 \Phi} V(\Phi)=1+4 \Phi_{N}+O\left(\Phi_{N}^{2}\right),
$$

with $\Phi_{N}=\Phi-\Phi_{\infty}$ for viable post-Newtonian parameters $\beta$ and $\gamma$. The equivalence of the gravitational and the inertial mass of the spherical gravitating body then holds. Note that (3.17) is equivalent to

$\frac{1}{V(\Phi)} \frac{\mathrm{d} V(\Phi)}{\mathrm{d} \Phi}=8 \quad$ at $\Phi=\Phi_{\infty}$.

Since a condition for the potential is obtained now, we also try to investigate the asymptotic gravitational field around an electric point charge in GSG. We introduce a point charge and consider the spherically symmetric solution. The Lagrangian density for the electromagnetic field is given by
$\mathcal{L}_{E M}=-\frac{1}{16 \pi} F_{\mu v} F^{\mu v}$,

where $F_{\mu \nu} \equiv \partial_{\mu} A_{v}-\partial_{v} A_{\mu}$. The energy-momentum tensor obtained from this Lagrangian is

$T_{\mu v}=-\frac{1}{4 \pi}\left(F_{\mu v}^{2}-\frac{1}{4} F^{2} q_{\mu v}\right)$,

where $F_{\mu \nu}^{2} \equiv F_{\mu \rho} F_{v \sigma} q^{\rho \sigma}$ and $F^{2} \equiv F_{\mu v}^{2} q^{\mu v}$.

A spherically symmetric solution for the equation of motion $\nabla_{\mu} F^{\mu v}=0$ is given by

$F_{0 r}=\frac{Q}{r^{2}} \sqrt{B(r) A(r)}$,

where $Q$ is a constant corresponding to the electric charge and we use the standard metric (3.3). Then we find

$T=0, \quad E=\frac{1}{8 \pi} \frac{Q^{2}}{r^{4}}, \quad C^{\lambda}=0$,

in $\chi$ (2.18) in the right-hand side of the equation for the scalar field (2.17). The equation of motion for the scalar field $\Phi(r)$ becomes

$$
\sqrt{\frac{V(\Phi(r))}{A(r) B(r)}} \frac{1}{r^{2}} \frac{\mathrm{d}}{\mathrm{d} r}\left[\sqrt{\frac{B(r)}{A(r)}} r^{2} \frac{\mathrm{d} \Phi(r)}{\mathrm{d} r}\right]=\frac{\kappa}{16 \pi}\left(2-\frac{1}{2 V} \frac{\mathrm{d} V}{\mathrm{~d} \Phi}\right) \frac{Q^{2}}{r^{4}},
$$

In the asymptotic region of large $r$, using (3.17), we can approximate the equation as

$\sqrt{\frac{V\left(\Phi_{\infty}\right)}{A(r) B(r)}} \frac{1}{r^{2}} \frac{\mathrm{d}}{\mathrm{d} r}\left[\sqrt{\frac{B(r)}{A(r)}} r^{2} \frac{\mathrm{d} \Phi(r)}{\mathrm{d} r}\right] \approx-\frac{\kappa}{8 \pi} \frac{Q^{2}}{r^{4}}$,

and we can also find an approximate solution

$B(r)=1-\frac{2 G M}{r}-\frac{G Q^{2}}{r^{2}}+O\left((G M / r)^{3}\right)$.

Unfortunately, this does not coincide with the ReissnerNordström solution in GR, because of the wrong sign in front of the $r^{-2}$ term. We should note that there is no reason to match exactly the solutions to GSG and GR.

Although we do not pursue the charged solution in GSG further in this paper, we suppose that the presence of nonminimal couplings between the vector and scalar fields could change the asymptotic behavior and it will be worth studying this possibility.

\section{A static spherical vacuum solution and singularity}

As we have seen in the previous section, the vacuum solution with spherical symmetry satisfies 


$$
\sqrt{\frac{b(r)}{A(r)}} r^{2} \frac{\mathrm{d} \Phi}{\mathrm{d} r}=\frac{1}{2} \frac{1}{\sqrt{A(r) b(r)}} r^{2} \frac{\mathrm{d} b(r)}{\mathrm{d} r}=G M e^{\Phi_{\infty}}>0
$$

where $b(r) \equiv e^{2 \Phi(r)}$ and $A(r)$ has been defined as (3.5). In this section, we start with undetermined asymptotic value for the scalar, $\Phi_{\infty}$. The Eq. (4.1) means that $q_{00}=B(r)=$ $b(r) e^{-2 \Phi_{\infty}}$ is a monotonically increasing function of $r$. Moreover, if the value of $b$ is positive everywhere, the above equation can be rewritten as

$$
\frac{\sqrt{V(\Phi(r)) / b(r)}}{\left|2 b(r)-r b^{\prime}(r)\right|} r^{2} b^{\prime}(r)=G M e^{\Phi_{\infty}}
$$

where the prime $\left(^{\prime}\right)$ stands for the derivative with respect to $r$.

If we assume that $b>0$ and $\sqrt{V(\Phi(r)) / b(r)}$ is finite in the vicinity of $r=0$, we see that

$\frac{b^{\prime}(r)}{b(r)} \rightarrow \infty \quad$ as $\quad r \rightarrow 0$.

(and also $\Phi^{\prime}(r) \rightarrow \infty$ ) from Eq. (4.2). As far as we assume a non-singular potential $\left(V(\Phi)<\infty\right.$ for $\left.-\infty<\Phi \leq \Phi_{\infty}\right)$ and non-singular metric $q_{00} \propto b$ for $r>0$, the vacuum solution has a singularity at the origin. This fact might bring about a possibility that a naked singularity emerges after a possible gravitational collapse. ${ }^{2}$ The difficulty of obtaining non-singular vacuum solution forces us to examine the solution of which metric becomes singular at finite $r$, which can be regarded as a horizon radius. ${ }^{3}$ If we set $b\left(r_{g}\right)=0$ and $b^{\prime}\left(r_{g}\right) \neq 0$ at the radius (of infinite-redshift) $r=r_{g}>0$, Eq. (4.1) tells us that

$0<\lim _{r \rightarrow r_{g}} \sqrt{A(r) b(r)}<\infty$.

This condition is a necessary condition that the radius $r=$ $r_{g}$ can be the horizon radius. Then, because $\lim _{r \rightarrow r_{g}} \Phi(r)=-\infty$, it should be satisfied that

$0<\lim _{\Phi \rightarrow-\infty} \sqrt{V(\Phi) / b(r)}<\infty$.

Further observation leads to that fact that the specific combination $2-\frac{r b^{\prime}}{b}$ in (4.2), which comes from $A(r)$, becomes negative at $r=r_{g}$. Since this must be positive for a large $r$, we conclude that the quantity $2-\frac{r B^{\prime}}{B}$ vanishes at a certain radius $r=r_{0}>r_{g}$. This condition requires

$V\left(\Phi\left(r_{0}\right)\right)=0$,

\footnotetext{
2 The time-dependent dynamics in GSG has not been studied, however. We should study the gravitational collapse in GSG.

3 The existence of naked singularity in nature is still controversial, however. For example, see Ref. [18-20].
}

because the both sides of (4.2) should be finite. Therefore the differential equation (4.2) for $b(r)$ is rewritten as

$$
\frac{r}{2} \frac{b^{\prime}(r)}{b(r)}=\left(1-r \frac{\sqrt{V(\Phi(r)) / b(r)}}{G M e^{\Phi_{\infty}}}\right)^{-1} \quad \text { for } r_{g}<r<r_{0}
$$

$$
\frac{r}{2} \frac{b^{\prime}(r)}{b(r)}=\left(1+r \frac{\sqrt{V(\Phi(r)) / b(r)}}{G M e^{\Phi_{\infty}}}\right)^{-1} \quad \text { for } r>r_{0},
$$

In order to obtain the smooth function (such as $b(r) \in C^{\infty}$ ), it should be assumed that

$V(\Phi) / b=[F(b)]^{2}$,

and $F(b)$ has a vanishing point, according to Eq. (4.6). Then the horizon radius $r_{g}$ is determined as

$\frac{G M e^{\Phi_{\infty}}}{r_{g}}=|F(0)| \equiv F_{0}$.

We will examine the case that the equation has exact analytic solutions. In the simplest case, $F$ is linear in $b$ such that

$$
F(b)=F_{0}\left(1-f_{1} b\right),
$$

where $F_{0}$ and $f_{1}$ are positive constants. Adopting a new variable $y \equiv 1-r_{g} / r$, the differential equation for $b(y)$ becomes

$\frac{\mathrm{d} b}{\mathrm{~d} y}=\frac{2 b}{-y+1-F(b) / F_{0}}=\frac{2 b}{-y+f_{1} b}$,

and the boundary condition is $b(0)=0$. The solution is

$b=\frac{3}{f_{1}} y=\frac{3}{f_{1}}\left(1-\frac{r_{g}}{r}\right)$.

Then, we find $e^{2 \Phi_{\infty}}=b(1)=\frac{3}{f_{1}}$ and

$$
\begin{aligned}
& B(r)=e^{2\left(\Phi-\Phi_{\infty}\right)}=1-\frac{r_{g}}{r}, \\
& A(r)=\frac{3}{4 F_{0}^{2} f_{1}}\left(1-\frac{r_{g}}{r}\right)^{-1} .
\end{aligned}
$$

To obtain asymptotic flat spacetime, we must choose

$F_{0}^{2}=\frac{3}{4 f_{1}}$.

Then, remembering $b=e^{2 \Phi}$, the scalar potential has the form

$$
\begin{aligned}
V(\Phi) & =\frac{3}{4 f_{1}} e^{2 \Phi}\left(1-f_{1} e^{2 \Phi}\right)^{2} \\
& =\frac{e^{4 \Phi_{\infty}}}{4} e^{2\left(\Phi-\Phi_{\infty}\right)}\left[1-3 e^{2\left(\Phi-\Phi_{\infty}\right)}\right]^{2},
\end{aligned}
$$


and $V\left(\Phi_{\infty}\right)=e^{4 \Phi_{\infty}}$. Thus, the post-Newtonian condition (3.17) is satisfied. The spacetime is the Schwarzschild spacetime, and thus $\beta=\gamma=1$. The Newton limit also tells $r_{g}=2 G M$, where $G=\kappa /\left(8 \pi \sqrt{V\left(\Phi_{\infty}\right)}\right)=f_{1} \kappa /(24 \pi)$. The potential (4.17) is essentially the Novello's potential $V_{\mathrm{N}}(\Phi)$, provided that $f_{1}=3$ (which implies $\Phi_{\infty}=0$ ).

Incidentally, choosing the function $F(b)$ as

$F(b)=F_{0}\left(1-f_{p} b^{p}\right)$

where $f_{p}$ is a constant, the differential equation for $b(y)$ becomes

$\frac{\mathrm{d} b}{\mathrm{~d} y}=\frac{2 b}{-y+f_{p} b^{p}}$

and then the equation is analytically solved as

$b=\left(\frac{2 p+1}{f_{p}}\right)^{1 / p} y^{1 / p}=\left(\frac{2 p+1}{f_{p}}\right)^{1 / p}\left(1-\frac{r_{g}}{r}\right)^{1 / p}$

and $e^{2 \Phi_{\infty}}=\left(\frac{2 p+1}{f_{p}}\right)^{1 / p}$. The components of the metric are then

$B(r)=e^{2\left(\Phi-\Phi_{\infty}\right)}=\left(1-\frac{r_{g}}{r}\right)^{1 / p}$,

$A(r)=\frac{1}{4 p F_{0}^{2}}\left(\frac{2 p+1}{f_{p}}\right)^{1 / p}\left(1-\frac{r_{g}}{r}\right)^{-2+1 / p}$.

If we choose $\frac{1}{4 p F_{0}^{2}}\left(\frac{2 p+1}{f_{p}}\right)^{1 / p}=1$, the solution describes an asymptotically flat spacetime, i.e.,

$\mathrm{d} s^{2}=\left(1-\frac{r_{g}}{r}\right)^{1 / p} \mathrm{~d} t^{2}-\left(1-\frac{r_{g}}{r}\right)^{-2+1 / p}-r^{2} \mathrm{~d} \Omega^{2}$.

The location $r=r_{g}$ is, however, a singularity of the metric (4.23), which comes from the fact that $\mathrm{d} b /\left.\mathrm{d} r\right|_{r=r_{g}}$ does not take a finite value (i.e., $b^{\prime}(0)=0$ or $b^{\prime}(0)=\infty$, where $b^{\prime}(y)$ is the first derivative of $\left.b(y)\right)$.

The physical condition $\beta=\gamma$ adopted in the previous section, i.e., the absence of $1 / r^{2}$ term in the expansion of $B(r)$ in terms of powers of $1 / r$, is transformed as $\lim _{y \rightarrow 1} b^{\prime \prime}(y)=0$, where $b^{\prime \prime}(y)$ is the second derivative of $b(y)$. Moreover, we can rewrite the condition (3.18), using the differential equation for $b(y)$, as

$2 b(1) F^{\prime}(b(1))=3 F(b(1))$,

where $F^{\prime}(b)$ is the first derivative of $F(b)$.

We further quest another possible potential, which leads to the existence of a horizon as well as the nice behavior at weak gravity, $\beta=\gamma=1$. We assume, with constants $f_{1}, f_{2}$ and $f_{3}$,

$$
F(b)=F_{0}\left(1-f_{1} b-f_{2} b^{2}-f_{3} b^{3}\right) .
$$

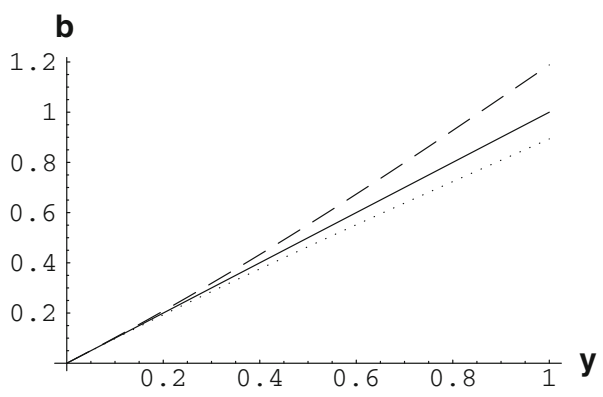

Fig. 1 The solutions for $b(y)$ are plotted against $y$. The rigid line exhibits the case with $f_{1}=3, f_{2}=0$ and $f_{3}=0$. The dotted line exhibits the case with $f_{1}=3, f_{2}=1$ and $f_{3} \approx-0.52226$. The broken line indicates the case with $f_{1}=3, f_{2}=-1$ and $f_{3} \approx 0.39273$

To satisfy $\lim _{y \rightarrow 1} b^{\prime \prime}(y)=0$, we must choose a set of parameters, $f_{1}, f_{2}$ and $f_{3}$. A set of parameters can be found numerically as $f_{1}=3, f_{2}=1$ and $f_{3} \approx-0.52226$. Then, $b(1) \approx 0.893544$ and $F_{0} \approx 0.448751$. Therefore, the horizon radius is $r_{g} \approx 2.10645 G M$. Another set is $f_{1}=3, f_{2}=$ -1 and $f_{3} \approx 0.39273$. Then, $b(1) \approx 1.18827$. and $F_{0} \approx 0.601671$. The horizon radius is $r_{g} \approx 1.81175 G M$ in this case. The solutions for $b(y)$ are displayed in Fig. 1. We find that the horizon radius can become larger or smaller than $2 G M$ by selecting the parameters, i.e., the form of the scalar potential $V(\Phi)$.

\section{Inverse problems in GSG}

\section{An inverse problem on black holes}

By now, we have found the conditions for the scalar potential $V(\Phi)$. Vast ambiguity in the form of the potential remains with the conditions. In this section, we consider an 'inverse problem', which require the potential form to obtain a given simple metric component. We assume the simplest 'solution' which is permissible up to the post-Newtonian order,

$b=\frac{3}{f}\left[y+h\left(y^{2}-\frac{1}{3} y^{3}\right)\right]$,

where $f(>0)$ and $h(>-1)$ are constants. Since $y=1-r_{g} / r$, we have

$$
b=e^{2 \Phi}=e^{2 \Phi_{\infty}}\left[1-\frac{2 G M}{r}+\frac{4}{3} \delta \frac{(2 G M)^{3}}{r^{3}}\right],
$$

where

$$
\begin{aligned}
e^{2 \Phi_{\infty}} & =\frac{3}{f}\left(1+\frac{2}{3} h\right), \quad \delta=\frac{h}{4} \frac{\left(1+\frac{2}{3} h\right)^{2}}{(1+h)^{3}}, \\
\frac{r_{g}}{2 G M} & =\frac{e^{\Phi_{\infty}}}{2 F_{0}}=\frac{1+\frac{2}{3} h}{1+h}, \quad F_{0}^{2}=\frac{3}{4 f} \frac{(1+h)^{2}}{1+\frac{2}{3} h} .
\end{aligned}
$$


If we solve the Eq. (5.2) for $2 G M / r$, we find

$$
\begin{aligned}
& \frac{2 G M}{r}=\frac{1}{\sqrt{\delta}} \sin \left[\frac{1}{3} \arcsin \left\{3 \sqrt{\delta}\left(1-e^{-2 \Phi_{\infty}} b\right)\right\}\right] \quad \text { for } \delta>0 \\
& \frac{2 G M}{r}=\frac{1}{\sqrt{|\delta|}} \sinh \left[\frac{1}{3} \operatorname{arcsinh}\left\{3 \sqrt{|\delta|}\left(1-e^{-2 \Phi_{\infty}} b\right)\right\}\right] \\
& \quad \text { for } \delta<0 .
\end{aligned}
$$

The function $F(b)$ defined in the previous section can be rewritten as

$$
F(b)=F_{0}(1-f \varphi(b)),
$$

and then we find that the function $\varphi(b)$ satisfies $\varphi^{\prime}(0)=1$. Substituting the 'solution' into the equation of motion, we get

$f \varphi(b)=Y(b)+\frac{2 f b}{3\left[1+h\left(2 Y(b)-Y(b)^{2}\right)\right]}$,

where

$$
\begin{aligned}
Y(b)=1 & -\frac{2 \sqrt{1+h}}{\sqrt{h}} \sin \left[\frac { 1 } { 3 } \operatorname { a r c s i n } \left\{\frac{3 \sqrt{h}\left(1+\frac{2}{3} h\right)}{2(1+h)^{3 / 2}}\right.\right. \\
& \left.\left.\left.\times 1-\frac{f}{3+2 h} b\right)\right\}\right] \quad \text { for } h>0, \\
Y(b)=1 & -\frac{2 \sqrt{1+h}}{\sqrt{|h|}} \sinh \left[\frac { 1 } { 3 } \operatorname { a r c s i n h } \left\{\frac{3 \sqrt{|h|}\left(1+\frac{2}{3} h\right)}{2(1+h)^{3 / 2}}\right.\right. \\
& \left.\left.\times\left(1-\frac{f}{3+2 h} b\right)\right\}\right] \quad \text { for } h<0 .
\end{aligned}
$$

In the limit of $h \rightarrow 0$, we obtain $f \varphi(b)=f b$ as we have already known. On the other hand, another limiting case $h \rightarrow \infty$ yields, if we set $f=3+2 h$ for further simplicity, $r_{g}=\frac{4 G M}{3}$ and one can find

$b=1-\frac{2 G M}{r}+\frac{4}{27} \frac{(2 G M)^{3}}{r^{3}}=1-\frac{3 r_{g}}{2 r}+\frac{r_{g}^{3}}{2 r^{3}}=\frac{3}{2} y^{2}-\frac{1}{3} y^{3}$.

The shape of the potential is shown in Fig. 2. Unfortunately the potential varies very slightly with $h$ in the present assumption for parametrization.

\section{An inverse problem on a spherical body: pseudo- gravastar}

Next, we consider a spherical configuration of a gravitating perfect fluid. We assume that the outside of the radius of such a 'star' is empty vacuum. The vacuum solutions and

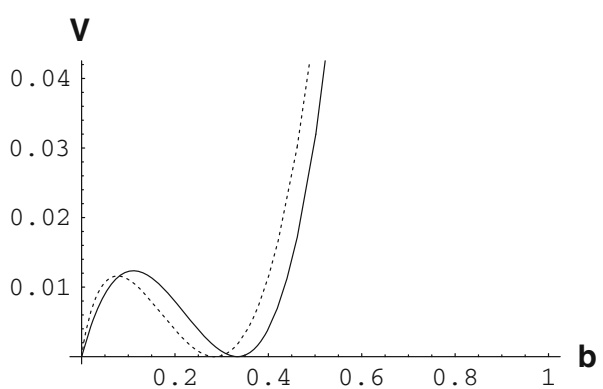

Fig. 2 The shape of the scalar potential is plotted against $b=e^{2 \Phi}$ (only shown for small $b$ ). The rigid line exhibits the case with $h=0$. The dotted line exhibits the case with $h=\infty$

the corresponding scalar potential have been assumed and found in the previous subsection.

We assume that the energy-momentum tensor is given in the form of a perfect fluid

$T_{\mu \nu}=(\rho+p) u_{\mu} u_{v}-p q_{\mu v}$,

where $u_{\mu}$ is the four-velocity which satisfies $q_{\mu \nu} u^{\mu} u^{v}=1, \rho$ is the energy density and $p$ is the pressure of the fluid. In the static case, the four-velocity has only the time-like component. Then, the quantities in (2.12) read

$$
T=\rho-3 p, \quad E=-p, \quad C^{\lambda}=0 .
$$

The dynamical Eq. (2.17) is expressed, with the metric (3.3), as

$$
\begin{aligned}
& \sqrt{\frac{V(\Phi(r))}{A(r) B(r)}} \frac{1}{r^{2}} \frac{\mathrm{d}}{\mathrm{d} r}\left[\sqrt{\frac{B(r)}{A(r)}} r^{2} \frac{\mathrm{d} \Phi(r)}{\mathrm{d} r}\right] \\
= & \frac{V(\Phi)}{r^{2} e^{3 \Phi}\left|1-r \Phi^{\prime}\right|}\left[\frac{\sqrt{V(\Phi)} r^{2} \Phi^{\prime}}{e^{\Phi}\left|1-r \Phi^{\prime}\right|}\right]^{\prime}=\frac{\kappa}{2}\left[\rho(r)-\left(5-\frac{1}{2 V} \frac{\mathrm{d} V}{\mathrm{~d} \Phi}\right) p(r)\right] .
\end{aligned}
$$

where the prime ' denotes the derivative with respect to the radial coordinate $r$. On the other hand, the conservation of the energy-momentum tensor leads to the equation

$$
\nabla_{\mu} T^{\mu v}=\frac{1}{\sqrt{-q}} \partial_{\mu}\left(\sqrt{-q} T^{\mu v}\right)+\Gamma_{\rho \sigma}^{v} T^{\rho \sigma}=0
$$

where

$$
\Gamma_{\rho \sigma}^{v}=\frac{1}{2} q^{\nu \lambda}\left(\partial_{\rho} q_{\lambda \sigma}+\partial_{\sigma} q_{\lambda \rho}-\partial_{\lambda} q_{\rho \sigma}\right) .
$$

For the perfect fluid, the equation of conservation becomes

$p^{\prime}(r)+[\rho(r)+p(r)] \Phi^{\prime}(r)=0$.

The Eqs. (5.14) and (5.17), together with a certain equation of state, which gives the relation between $\rho$ and $p$, completely governs the structure of the spherical body in GSG. This is a direct problem on the structure of a 'star'. 
Solving the equations is straightforward but tedious in genaral cases. We leave the study on the generic solutions for various equation of state for another separate work. Here, we assume, the smooth metric inside the 'star' and we investigate the behaviors of the energy density and the pressure as another inverse problem.

In this study, we simply assume that the (00) component of the metric inside a spherical star can be expressed by

$$
q_{00}=C_{0}-C_{2} r^{2}
$$

where $C_{0}$ and $C_{2}$ are constants. Incidentally, this behavior matches the de Sitter space in GR. Mazur and Mottla proposed 'gravastars', which have the de Sitter spacetime structure inside them, about a decade ago [21-24]. Therefore, the present analysis can be considered as the first step to investigate such simple and interesting exotic objects, say, 'pseudo-gravastars' in GSG (and similar theories mentioned in "Introduction").

Although the gravastars in GR may have a shell of an exotic matter at their surface, we take a simple assumption in this paper, for the 'pseudo-gravastars' in GSG; We define the surface of a star as $p\left(r_{*}\right)=0$, where $r_{*}$ is the radius of the star.

We use the result of the previous subsection, so we adopt $V(\Phi)=e^{2 \Phi} F\left(e^{2 \Phi}\right)^{2}$, where $F$ is defined by (5.7)(5.10) with the choice $f=3+2 h$. The metric for the exterior of the star can be described by

$$
\begin{aligned}
b(r)= & e^{2 \Phi(r)}=b_{e}(r)=1-\frac{2 G M}{r}+\frac{4 \delta}{3} \frac{(2 G M)^{3}}{r^{3}}, \\
& \text { for } r>r_{*} .
\end{aligned}
$$

On the other hand, we assume that the metric is given by the function (5.18) which smoothly connects to $b_{e}(r)$ :

$b(r)=b_{*}(r) \equiv b_{e}\left(r_{*}\right)-\frac{b_{e}^{\prime}\left(r_{*}\right)}{2}\left(1-\frac{r^{2}}{r_{*}^{2}}\right) \equiv e^{2 \Phi_{*}(r)}$,

$$
\text { for } r<r_{*} \text {. }
$$

For $r<r_{*}$, eliminating $\rho$ from Eqs. $(5.14,5.17)$ yields

$$
\begin{aligned}
& {\left[e^{6 \Phi_{*}(r)} \frac{1}{\sqrt{V\left(\Phi_{*}(r)\right)}} \kappa p(r)\right]^{\prime}=} \\
& -\frac{2 e^{3 \Phi_{*}(r)} \sqrt{V\left(\Phi_{*}(r)\right)} \Phi_{*}^{\prime}(r)}{r^{2}\left(1-r \Phi_{*}^{\prime}(r)\right)}\left[\frac{\sqrt{V\left(\Phi_{*}(r)\right)} r^{2} \Phi_{*}^{\prime}(r)}{e^{\Phi_{*}(r)}\left(1-r \Phi_{*}^{\prime}(r)\right)}\right]^{\prime},
\end{aligned}
$$

for $r<r_{*}$. To obtain the solution for $p(r)$, we have only to integrate the equation.

Some numerical examples are shown in Figs. 3, 4, 5 and 6. In each figure, $10 \kappa r_{g}^{2} p$ and $\kappa r_{g}^{2} \rho$ are plotted against $r / r_{g}$. Figures 3 and 4 are in the case with the parameter $h=0$ while Figs. 5 and 6 are in the case with $h=\infty$. In Figs. 3

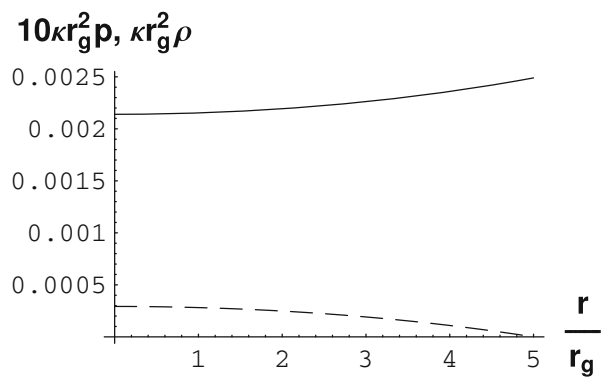

Fig. 3 The energy density (solid line) and the pressure (dashed line) are plotted against $r / r_{g}$ in the case with the parameter $h=0$ and $r_{*}=5 r_{g}$

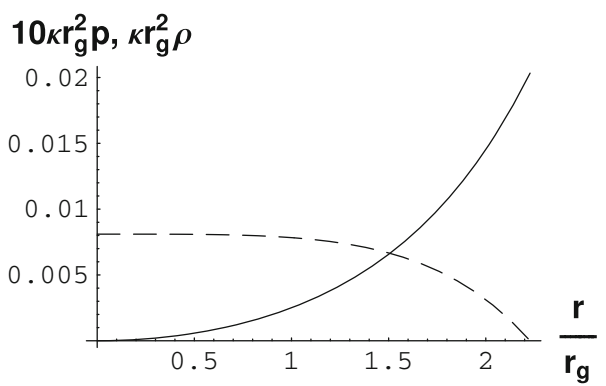

Fig. 4 The energy density (solid line) and the pressure (dashed line) are plotted against $r / r_{g}$ in the case with the parameter $h=0$ and $r_{*}=2.228 r_{g}$

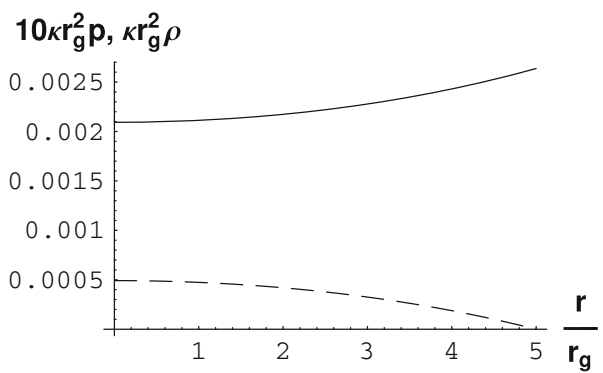

Fig. 5 The energy density (solid line) and the pressure (dashed line) are plotted against $r / r_{g}$ in the case with the parameter $h=\infty$ and $r_{*}=5 r_{g}$

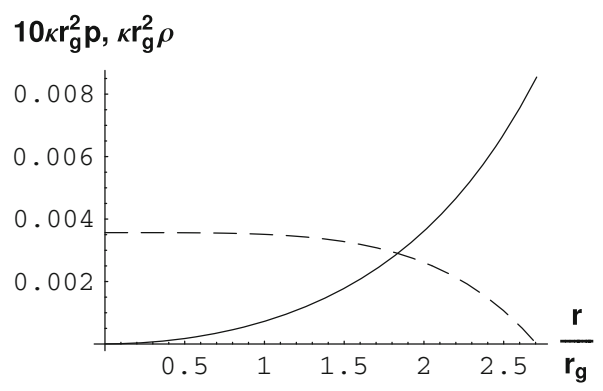

Fig. 6 The energy density (solid line) and the pressure (dashed line) are plotted against $r / r_{g}$ in the case with the parameter $h=\infty$ and $r_{*}=2.707 r_{g}$ 
and 5 , we choose $r_{*}=5 r_{g}$. Figures. 4 and 6 indicate the results with $\rho(0)=0$.

In both cases of $h=0$ and $h=\infty$, the behaviors of $\rho$ and $p$ are qualitatively equal, respectively. In Figs. 4 and 6, the values for $r_{*}$ are considered as the lower bound for the radii of the stars of the present type. Below the critical value for the radius, the energy density at the origin becomes negative.

In contrast to the gravastars in GR, we find that the value of the pressure $p$ can be positive and simply decreasing in the present pseudo-gravastar in GSG. Of course, the geometry of the interior of the star is not an exact de Sitter space, for $q_{11} \neq-\left(q_{00}\right)^{-1}$ in GSG.

The 'stars' constructed above are, however, not realistic because the energy density $\rho$ also should decrease along with $r$ for a normal equation of state. ${ }^{4}$ Nevertheless, we suppose that the characteristic scales of the 'star' in GSG revealed in the simple approach are useful for setting the starting values of numerical calculation.

\section{Summary and prospects}

In this paper, we considered the geometric scalar gravity of Novello and collaborators and found conditions of the scalar potential that had a permissible solution in the case of weak and strong gravity. We found that the simple potential of Novello et al. leads to the solution which agrees with the Schwarzschild solution of GR and small corrections to the potential are permitted and the generic potential leads to the 'black hole solution' with a modified mass-horizon radius relation (i.e., $r_{g} \neq 2 G M$ ), of which asymptotic behavior describes weak gravity up to the postNewtonian order. We also showed possible solutions for 'pseudo-gravastars' in GSG with generic scalar potentials.

We will try to extend the geometric scalar theory of gravity with additional degrees of freedom. We also wish to study the mass-radius relation (known in GR [25]) which may exist in a solution describing a spherical star in GSG by numerical analyses. In addition, we want to study 'cosmologies' expressed by the generic scalar potentials in geometric scalar gravity. We hope to report soon on these issues.

The quantization of GSG is another very interesting subject. We wish to discuss it in a separate paper.

\section{Note added:}

After completion of this manuscript, we have noticed a new preprint arXiv:1508.02665 [gr-qc] by Jardim and Landim [26] on GSG. It discusses the issue of the

\footnotetext{
4 As for a possible improvement, we should take shell boundaries and anisotropic pressures for exotic stars into account.
}

cosmological constant in GSG and argues an inverse problem' to realize the de Sitter spacetime in GSG.

Acknowledgments We thank Prof. Kumar Shwetketu Virbhadra for valuable information on naked singularities. We also thank Prof. Sunny Vagnozzi for providing information on their work on modified gravity including scalar derivative terms $[27,28]$.

Open Access This article is distributed under the terms of the Creative Commons Attribution 4.0 International License (http://crea tivecommons.org/licenses/by/4.0/), which permits unrestricted use, distribution, and reproduction in any medium, provided you give appropriate credit to the original author(s) and the source, provide a link to the Creative Commons license, and indicate if changes were made.

\section{References}

1. Nojiri, S., Odintsov, S.D.: Phys. Rep. 505, 59 (2011)

2. Capozziello, S., De Laurentis, M.: Phys. Rep. 509, 167 (2011)

3. Clifton, T., Ferreira, P.G., Padilla, A., Skordis, C.: Phys. Rep. 513, 1 (2012)

4. Novello, M., Bittencourt, E., Moschella, U., Goulart, E., Salim, J.M., Toniato, J.D.: Geometric scalar theory of gravity, JCAP 1306 (2013) 014; JCAP 1401 (2014) 01, E01

5. Novello, M., Bittencourt, E.: The gravitational energy. arXiv: 1311.6984 [gr-qc] (2013)

6. Bittencourt, E., Moschella, U., Novello, M., Toniato, J.D.: Cosmology in geometric scalar gravity. Phys. Rev. D90, 123540 (2014)

7. Bittencourt, E., Novello, M., Moschella, U., Goulart, E., Salim, J.M., Toniato, J.D.: Geometric scalar gravity. Nonlinear Phenom. Complex Syst. 17, 349 (2014)

8. Toniato, J.D.: A teoria geométrica-escalar da gravitação e sua aplicação à cosmologia, Tese de Doutorado, Rio de Janeiro (2014)

9. Kraichman, R.: Phys. Rev. 98, 1118 (1955)

10. Freund, P.G.O., Nambu, Y.: Phys. Rev. 174, 1741 (1968)

11. Deser, S., Halpern, L.: Gen. Rel Grav. 1, 131 (1970)

12. Shapiro, S.L., Teukolsky, S.A.: Phys. Rev. D47, 1529 (1993)

13. Novello, M.: Talk in hot topics in modern cosmology, spontaneous workshop IX, 27 April-1 May 2015, Cargèse

14. Chamseddine, A.H., Mukhanov, V.: JHEP 1311, 135 (2013)

15. Bekenstein, J.D.: Phys. Rev. D70, 083509 (2004)

16. Weinberg, S.: Gravitation and Cosmology, pp. 184. Wiley, New York (1972)

17. Hartle, J.B.: Gravity, An Introduction to Einstein's General Relativity, pp. 222. Addison Wesley, New York (2003)

18. Claudel, C.-M., Virbhadra, K.S., Ellis, G.F.R.: J. Math. Phys. 42, 818 (2001)

19. Virbhadra, K.S., Ellis, G.F.R.: Phys. Rev. D65, 103004 (2002)

20. Virbhadra, K.S., Keeton, C.R.: Phys. Rev. D77, 124014 (2008)

21. Mazur, P.O., Mottola, E.: Proc. Natl. Acad. Sci. USA (PNAS) 101, 9545 (2004). arXiv: gr-qc/0109035

22. Cattoën, C., Faber, T., Visser, M.: Class. Q. Grav. 22, 4189 (2005)

23. Faber, T.: arXiv: gr-qc/0607029

24. DeBenedictis, A., Horvat, D., Ililić, S., Kloster, S., Viswanathan, K.S.: Class. Q. Grav. 23, 2303 (2006)

25. Buchdahl, H.A.: Phys. Rev. 116, 1027 (1959)

26. Jardim, I.C., Landim, R.R.: arXiv:1508.02665 [gr-qc]

27. Myrzakulov, R., Sebastiani, L., Vagnozzi, S.: Eur. Phys. J. C75, 444 (2015)

28. Myrzakulov, R., Sebastiani, L., Vagnozzi, S., Zerbini, S.: Fund. J. Mod. Phys. 8, 119 (2015) 